\title{
Caracterización temática de la investigación en Ciencia de la Información en Brasil en el período 2000-2009
}

\author{
Thematic characterization of research on \\ Information Science in Brazil \\ from 2000-2009
}

\author{
Gustavo LIBERATORE' \\ Víctor HERRERO-SOLANA²
}

\section{Resumen}

Este artículo tiene por objetivo analizar cuáles han sido los principales temas de interés en el campo de la investigación científica dentro del área de la Ciencia de la Información en Brasil a lo largo de la primera década de este siglo (2000-2009). Para ello se ha analizado el contenido de la producción científica publicada en cuatro de las principales revistas nacionales de la disciplina: Ciência da Informação, DataGramaZero, Perspectivas em Ciência da Informação y Transinformação. La metodología aplicada se basa en el análisis de co-ocurrencia de palabras-clave y en la representación e interpretación de los resultados por medio del análisis de redes sociales. Para dotar de un marco de interpretación a los resultados obtenidos se propone una comparación con las áreas temáticas propuestas por la Associação Nacional de Pesquisa e Pós-Graduação em Ciência da Informação en sus Grupos de Trabajo por tratarse de un espacio institucionalizado en donde, por consenso, se debate la investigación en la Ciencia de la Información brasileña. Se concluye en que es el estudio de la propia disciplina la que acapara los mayores esfuerzos de investigación.

Palabras-clave: Análisis de co-ocurrencias. Análisis de redes sociales. Brasil. Ciencia de la información. Investigación.

\begin{abstract}
The purpose of this article is to analyze which were the main topics of interest in the field of scientific research in Information Science during the first decade of the $21^{15 t}$ century in Brazil (2000-2009). In order to do this, the content of scientific production published in four of the most important national journals in the field was analyzed, namely: Ciência da Informação, DataGramaZero, Perspectivas em Ciência da Informação, and Transinformação. The methodology applied is based on co-occurrence analysis of keywords and the representation and interpretation of results by analyzing social networks. To provide a frame of interpretation of theresults obtained, the thematic areas proposed by the Working Groups of the Associação Nacional de Pesquisa e Pós-Graduação em Ciência da Informação were compared because it is an institutionalized space in which, by consensus, research on Information Science is discussed in Brazil. We conclude that the major research efforts are acquired when this field is studied.
\end{abstract}

Keywords: Co-word analysis. Social network analysis. Brazil. Information science. Research.

\footnotetext{
1 Universidad Nacional de Mar del Plata, Facultad de Humanidades, Departamento de Documentación. Funes3250, Mar del Plata, Argentina. Correspondencia a nombre de/Correspondence to: G. LIBERATORE.E-mail:<gliberat@mdp.edu.ar>.

2 Universidad de Granada, Facultad de Comunicación y Documentación, Departamento de Biblioteconomía y Documentación. Granada, España. Recebido el día 10/8/2012, re-presentado el 7/11/2012 y aceptado para su publicación el 9/1/2013.
} 


\section{Introducción}

El análisis de la investigación científica con base en la utilización de indicadores provenientes de fuentes de datos bibliográficas es un procedimiento clásico en la actualidad. A nivel internacional se han intensificado en las dos últimas décadas los estudios de campos disciplinares basados mayoritariamente en el análisis de los productos intelectuales generados por la investigación, apoyados en la aplicación del método bibliométrico, a través de la obtención de indicadores de tipo bibliográfico. Cada una de las variantes de análisis aplicadas son coincidentes en su objeto de estudio, es decir, se basan en lo que genéricamente se denomina "producción científica". Dentro de esta metodología se asume que la principal fuente de datos son las revistas científicas ya que constituyen uno de los principales canales de comunicación y difusión de los resultados de investigación (Maltrás-Barba, 2003).

Brasil ha desarrollado en las últimas décadas un vigoroso sistema científico que hoy lo sitúa en el primer plano a nivel regional y con una fuerte proyección internacional en términos de visibilidad. De la mano de este proceso virtuoso la Ciencia de la Información brasileña ha logrado insertarse dentro de las políticas científicas que han impulsado esta realidad emergiendo en la actualidad, tanto desde el punto de vista de la formación académica como de generación de la investigación, como un modelo a seguir por los países del continente.

La Ciencia de la Información en Brasil ha tenido un desarrollo y crecimiento muy importante en las últimas décadas, fruto de un proceso de institucionalización muy fuerte, tanto en lo profesional como en el ámbito académico/científico, al amparo de políticas públicas de fomento y regulación (Pinheiro; Loureiro, 2004; Kobashi, 2007; Eliel, 2008). En la actualidad es un área disciplinar definitivamente consolidada en el campo de las Ciencias Sociales y el crecimiento institucional ha estado de la mano de un temprano inicio en la formación de posgrado, un fuerte impulso a la investigación (Miranda; Barreto, 2000) y un fortalecimiento de los servicios de información que puedan solventar las actividades científicas y técnicas (Pinheiro, 2000). La producción científica generada por el país en este campo lidera holgadamente el ranking latinoamericano en esta materia - en volumen y visibilidad
-, fruto de haber obtenido en todo este tiempo de evolución y crecimiento una importante masa crítica de investigadores, docentes y profesionales. En este sentido, la Ciencia de la Información brasileña ha sido observada y comparada en varios estudios desde la óptica latinoamericana o iberoamericana destacándose su creciente performance en las últimas dos décadas (Licea de Arenas et al., 2000; Moya-Anegón; Herrero-Solana, 2002; Herrero-Solana; Ríos-Gómez, 2006; Herrero-Solana; Liberatore, 2008; Herrero-Solana; Miguel, 2010). Al mismo tiempo algunos estudios han marcado particularmente la tendencia a elegir espacios de divulgación nacionales por parte de los investigadores brasileños para la difusión de la producción científica (Noronha; Población, 2002; Parreiras et al., 2006; Pinto; Moreiro-González, 2010). Esta realidad responde en gran medida al hecho de que los canales de comunicación de las actividades generadas en el plano profesional y científico han venido multiplicándose sostenidamente en el tiempo, existiendo en la actualidad una veintena de títulos de publicaciones periódicas activas de la especialidad y un gran número de eventos nacionales y estaduales que se celebran con regularidad.

Dentro de este espectro, el aumento de los estudios empíricos es una consecuencia natural del desarrollo que la disciplina ha tenido frente a la necesidad de observar los contornos de un campo intelectual en permanente crecimiento. Así, las investigaciones realizadas no se concentran solamente en evaluar aquellos indicadores derivados de los productos intelectuales generados, sino también en las correlaciones existentes entre la formación de recursos humanos, las instituciones responsables y las políticas científicas existentes. Particularmente, para el objeto de estudio de este trabajo sesgado hacia los temas preponderantes de investigación, se cuentan numerosos antecedentes. Por un lado se ubican trabajos enfocados al análisis de alguna temática en particular (Mendonça, 2000; Francelin, 2004; Freire, 2008); por otro, aquellas investigaciones orientadas a explorar múltiples indicadores en los artículos publicados en una sola revista o fuente (Vieira, 1997; Mueller et al., 2000; Mueller; Pecegueiro, 2001; Silva, 2002; Pinheiro et al., 2005; Liberatore et al., 2007); finalmente y como antecedentes más cercanos y directos aparecen aquellos trabajos cuyo objetivo es indagar el comportamiento temático de la investigación 
en un período de tiempo suficientemente amplio (Pecegueiro, 2002; Pinheiro, 2005; Araújo; Melo, 2011).

El objetivo general de este trabajo es el de complementar y actualizar estos abordajes empíricos de la actividad científica a través de dos técnicas que han resultado ser muy eficaces y exitosas en términos de la representación de un campo intelectual como son el cálculo de co-ocurrencia y el Análisis de Redes Sociales (ARS). Más específicamente se trata de establecer cuáles son los frentes de investigación preponderantes, equipararlos con las áreas temáticas institucionalmente formalizadas por la Associação Nacional de Pesquisa e Pós- Graduação em Ciência da Informação (ANCIB) y compararlos con los resultados obtenidos en los estudios similares citados anteriormente.

\section{Métodos}

El abordaje de este tipo de análisis ha estado siempre sometido a debate en relación a cuáles son los métodos más adecuados para representar la constitución del dominio disciplinar desde su estructuración temática. Fundamentalmente la discusión se centra en los niveles de subjetividad subyacentes en el modelo de clasificación temática seleccionado para este tipo de representaciones (Jarvelin; Vakkari, 1990; Delgado-López-Cozar, 2002) y en la validez o actualidad de ciertos esquemas para visualizar la constitución del campo intelectual.

En este caso se ha decidido no adoptar un esquema preconfigurado para el análisis y representación del campo temático abordado por los artículos objeto de estudio. La razón principal de esta elección, de índole metodológica, se debe al hecho de evitar una interpretación forzada y arbitraria. En este sentido, se ha tomado como fuente de información a las palabras-clave asignadas por los autores en cada artículo lo cual genera, a nuestro entender, una representación más ajustada a la percepción que ellos mismos tienen del campo semántico.

Se han seleccionado para este análisis cuatro de las revistas nacionales del campo disciplinar más prestigiosas: Ciência da Informação editada por el Instituto Brasileiro de Informação em Ciência e Tecnologia (IBICT), DataGramaZero: Revista de Ciência da Informação editada por Instituto de Adaptação e Inserção na Sociedade da
Informação (IASI), Perspectivas em Ciência da Informação editada por la Escola de Ciência da Informação de la Universidade Federal de Minas Gerais (ECI/UFMG) y Transinformação editada por el Departamento de Programa de Pós-Graduação em Ciência da Informação de la Pontifícia Universidade Católica de Campinas (PUCCampinas). Los criterios de dicha selección se han basado, por un lado, en la calificación otorgada a esta publicaciones por el sistema Qualis de la Coordenação de Aperfeiçoamento de Pessoal de Nível Superior (Capes) que asigna para las revistas Ciência da Informação y Perspectivas em Ciência da Informação el valor de A2 (la más alta que han obtenido títulos de la disciplina) y para DataGramaZero y Transinformação el valor B2. Al mismo tiempo se tuvo en cuenta la visibilidad de las publicaciones medida en términos de las bases de datos que las indizan comprobando que las cuatro se hallan en los principales repertorios nacionales e internacionales de la especialidad. Incluso tres de ellas fueron incorporadas recientemente en las dos principales bases de datos multidisciplinares a nivel mundial como son el Institutefor Scientific Information (ISI) de Thomson Scientific (Perspectivas em Ciência da Informação y Transinformação desde 2008) y Scopus de Elsevier (Ciência da Informação desde 2006 y Perspectivas em Ciência da Informação desde 2008).

Desde el punto de vista temporal el lapso seleccionado es de diez años y se sitúa en el período 2000-2009.

El índice final quedó conformado por 2498 entradas (palabras-clave) pertenecientes a los 965 artículos procesados. Luego se procedió al análisis de este universo en base al cálculo de la co-ocurrencia (co-word analysis) de las mismas. Éste es un método basado en el concepto de similaridad asumiendo que, en la medida en que dos o más documentos co-ocurran, es decir, ocurran al mismo tiempo, parejas de expresiones conceptuales (palabras-clave en este caso), dichos documentos (artículos) poseen una similaridad o afinidad temática (Miguel et al., 2007). El cálculo del índice de parejas de palabras-clave se realizó a través del software Bibexcel tomando en cuenta las frecuencias más altas (Tabla 1) dentro de la distribución obtenida, fijándose un umbral de 8 ocurrencias para una representación suficientemente significativa.

La matriz de datos resultante fue representada y analizada por medio de una red social utilizando el 
Tabla 1. Palabras-clave con frecuencias más altas.

\begin{tabular}{|c|c|c|c|}
\hline Palabra-clave & Frecuencia & Palabra-clave & Frecuencia \\
\hline Ciência da informação & 114 & Disseminação da informação & 13 \\
\hline Informação & 82 & Interdisciplinaridade & 13 \\
\hline Gestão do conhecimento & 49 & Pesquisa & 13 \\
\hline Comunicação científica & 45 & Tecnologia & 13 \\
\hline Sociedade da informação & 40 & Bibliotecas universitárias & 12 \\
\hline Internet & 38 & Avaliação & 12 \\
\hline Conhecimento & 35 & Biblioteca digital & 11 \\
\hline Gestão da informação & 33 & Ciberespaço & 11 \\
\hline Bibliometria & 32 & Linguagem documentária & 11 \\
\hline Profissionais da informação & 32 & Representação do conhecimento & 11 \\
\hline Inteligência competitiva & 23 & Comunicação & 11 \\
\hline Brasil & 22 & Cidadania & 10 \\
\hline Produção científica & 21 & Biblioteca escolar & 10 \\
\hline Ontologias & 19 & Epistemologia & 10 \\
\hline Organização do conhecimento & 18 & Inclusão digital & 10 \\
\hline Sistemas de informação & 17 & Acesso livre & 9 \\
\hline Hipertexto & 16 & Educação & 9 \\
\hline Tecnologia da informação & 16 & Informetria & 9 \\
\hline Terminologia & 15 & Arquivos abertos & 9 \\
\hline Biblioteconomia & 15 & Competência informacional & 9 \\
\hline Organização da informação & 15 & Sociedade do conhecimento & 8 \\
\hline Bibliotecas digitais & 14 & Periódicos eletrônicos & 8 \\
\hline Indexação & 14 & Usabilidade & 8 \\
\hline Bibliotecário & 14 & Ensino & 8 \\
\hline Fontes de informação & 14 & Cienciometria & 8 \\
\hline Metadados & 14 & Globalização & 8 \\
\hline Recuperação da informação & 14 & & \\
\hline
\end{tabular}

Fuente: Elaboración propia (2012).

software Pajek. Para su representación se recurrió a un modelo vectorial procesado mediante el algoritmo de distribución espacial de Kamada-Kawai (1989).

Para valorar adecuadamente los componentes del grafo se han aplicado tres medidas de centralidad que son las más comúnmente utilizadas en el análisis de redes sociales: grado, intermediación y centralidad estructural (Hanneman; Riddle, 2005). Entendemos por grado de centralidad (Degree) al número de enlaces relacionados con un nodo y que permite determinar los actores más importantes de una red con respecto al resto. En el valor de intermediación (Betweenness) se expresa el nivel de influencia que ejerce un nodo en el contexto de la red y el control en el flujo de la información a través de todos los caminos que lo conectan a otros nodos o, en otras palabras, cuán involucrado está un nodo en la estructura de relaciones. La centralidad estructural o global (Eigenvector) tiene por objetivo ordenar la red en torno a las nociones de "cercanía" y "lejanía" de acuerdo a un orden natural entre el centro, el margen y la periferia de la gráfica resultante (Vargas-Quesada et al., 2010). La obtención de estos indicadores de la red fue por medio del software Ucinet 6 for Windows.

Finalmente y para dotar de un mejor marco de interpretación a los resultados obtenidos se propone una comparación de los tópicos emergentes de la red con las áreas temáticas propuestas por la Associação Nacional de Pesquisa e Pós-Graduação em Ciência da Informação (ANCIB) en sus Grupos de Trabajo (GT) por tratarse de un espacio institucionalizado en donde, por consenso, se debate la investigación en la Ciencia de la Información brasileña. Estos grupos están conformados en la actualidad en diez espacios de discusión (en el sitio de la ANCIB figura el grupo no 11 Informação \& Saúde pero aún no se han definido sus alcances): GT1: Estudos Históricose Epistemológicos da Ciência da Informação; GT2: Organização e Representação do Conhecimento; GT3: Mediação, Circulação e Apropriação da Informação; GT4: 
Gestão da Informação e do Conhecimento nas Organizações; GT5: Política e Economia da Informação; GT6: Informação, Educação e Trabalho; GT7: Produção e Comunicação da Informação em CT\&l; GT8: Informação e Tecnologia; GT9: Museu, Patrimônio e Informação; GT10: Informação e Memória.

\section{Resultados y Discusión}

A partir del cálculo de co-ocurrencia de las expresiones con mayor frecuencia se obtuvo una matriz de datos que fue representada y analizada por medio de una red social. La misma quedó conformada por 53 nodos y sus relaciones, sobre los cuales se aplicaron las medidas de centralidad antes mencionadas (Tabla 2). Se han añadido al grafo las siglas de los grupos de trabajo de la ANCIB como demarcación de los grupos temáticos observados y que actúan en esta representación como los componentes principales de la red (Figura 1).

De acuerdo a las medidas de centralidad calculadas seis nodos emergen con mayor gravitación en la red representando las áreas temáticas más desarrolladas y de mayor interés en este período. Estos son: Ciência da Informação, Informação, Gestão do Conhecimento, Comunicação Científica, Internet y Sociedade da Informação.

En la imagen de la red los nodos con mayor centralidad han sido señalados con diferente color para

Tabla 2. Nodos con mayor centralidad de la red social de co-ocurrencia de palabras-clave.

\begin{tabular}{lccc}
\hline Tema & Grado & Intermediación & Centralidad estructural \\
\hline Ciência da Informação & 40 & 412862 & 0.350 \\
Informação & 31 & 182293 & 0.299 \\
Gestão do Conhecimento & 23 & 98896 & 0.225 \\
Comunicação Científica & 22 & 84925 & 0.222 \\
Internet & 22 & 100088 & 0.211 \\
Sociedade da Informação & 20 & 47349 & 0.216 \\
\hline
\end{tabular}

Fuente: Elaboración propia (2012).

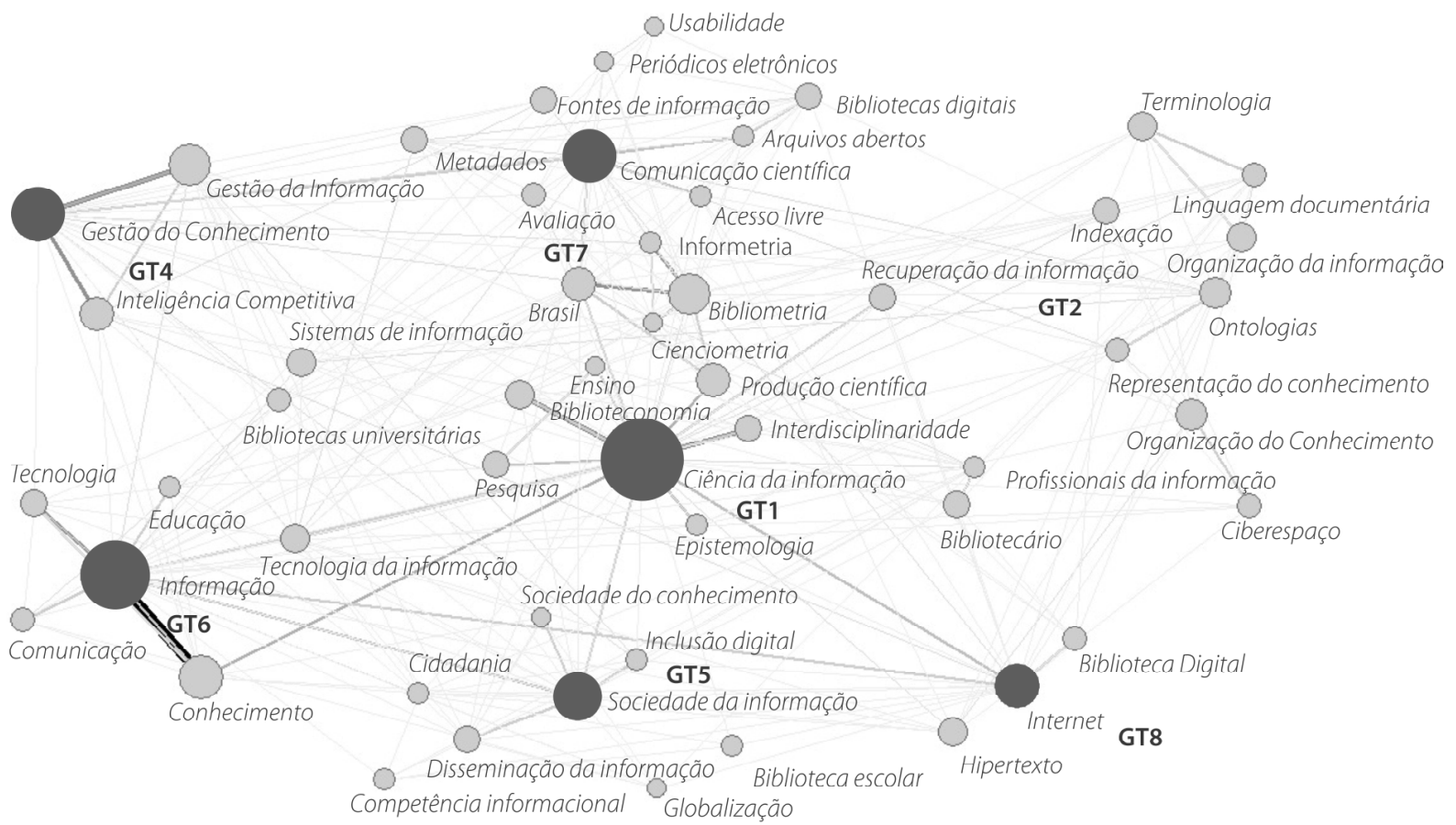

Figura 1. Red social de co-ocurrencia de palabras-clave.

Fuente: Elaboración propia (2012). 
una mejor visualización y se han añadido las siglas de los grupos temáticos designados por la ANCIB que guardan equivalencia no sólo con los nodos centrales sino también con otros agrupamientos de la red.

La primera observación sobre la resultante de este análisis es que la red se presenta como un gran bloque de relaciones entre los nodos - algunas de ellas muy fuertes y otras muy débiles -, sin la visualización de sub redes, lo que indica a priori que la producción científica estudiada no presenta ámbitos claramente definidos en cuanto a su especialidad temática. No obstante ello, no debe perderse de vista el hecho de que la representación analizada está sujeta al modo en que los investigadores caracterizan sus publicaciones, aspecto éste atravesado por componentes relativos a la subjetividad, al nivel de consenso alcanzado en la terminología empleada por la comunidad científica y a la dispar densidad (cantidad) de expresiones utilizadas en la descripción de los artículos.

Adentrándonos en el análisis particular de la red el nodo Ciência da Informação (GT1) aparece como el de mayor centralidad asociado fuertemente a cuestiones ligadas a la enseñanza, la investigación y la producción científica a nivel nacional. Es interesante observar además la aparición de tópicos que dan cuenta del interés por aspectos relativos a los procesos de institucionalización cognitiva de la disciplina como lo son la Interdisciplinaridade y los abordajes desde la Epistemologia. Resultan evidentes también los fuertes lazos que mantiene con otros nodos que, de acuerdo a la posición central que ocupan, pueden ser considerados como temáticas de fuerte interés en la investigación. La primera de ellas es la Informação (GT6) con un vínculo muy marcado al Conhecimento y tópicos conexos como la Comunicação, la Educação y las Tecnologías. Este agrupamiento sesgado fuertemente por el tándem Informação-Conhecimento aparece como muy abarcativo, de difícil delimitación en términos de cuáles son las líneas preponderantes de trabajo. Esto es así porque en torno a estos temas se encolumnan artículos de fuerte contenido teórico de diferentes vertientes, atendiendo una gama de problemáticas que van desde situar a la Informação como objeto de estudio hasta el análisis de aspectos relativos al campo laboral y el perfil profesional.

La otra temática de importancia vinculada a la Ciência da Informação es el de la Sociedade da Informação
(GT5) en una relación que por lo general se funda en la mirada que se tiene de este fenómeno desde el campo disciplinar bajo una vertiente paradigmática. No obstante ello, estamos en presencia de un campo relativamente autónomo que ha surgido a partir de la aparición de políticas públicas en Brasil en torno a lo que se ha dado en llamar el "Programa para la Sociedad de la Información" y que se ha visto materializado con la publicación del "Libro Verde" por parte del Ministério da Ciência e Tecnologia en el año 2000. Los tópicos frecuentados en este espacio son los asociados a la Globalização, la Inclusão digital, la Sociedade do Conhecimento y la Disseminação de Informação entendida desde las políticas de acceso.

Otro de los ámbitos que despierta mayor interés en el campo de la investigación es el relativo a la Comunicação Científica (GT7), tema que aparece vinculado a una serie de tópicos que representan diferentes líneas de trabajo. Por un lado las referidas a las formas y soportes de la comunicación: Periódicos eletrônicos, Fontes de informação, Bibliotecas digitais, y Arquivos abertos. Por otro, las que se circunscriben a un aspecto que cobró enorme importancia en la primera década de este siglo como lo es el Acesso livre a la información científica y técnica. También aparecen reflejadas las cuestiones relativas a la Avaliação traducida fundamentalmente en los siguientes aspectos: la evaluación de pares, la de las fuentes de información y la de la producción científica.

Dentro de este agrupamiento se destacan de manera muy significativa los tópicos relativos a los métodos bibliométricos aunque, dado el valor de centralidad que poseen los nodos que lo componen (Bibliometria, Cienciometria e Informetria), pueden dimensionarse como un sub agrupamiento que guarda cierta independencia en sus relaciones, como un campo de investigación con cierta autonomía. Así, se observa que estructuralmente los principales vínculos que conectan a este conjunto son la Comunicação Científica y la Produção Científica.

En otro orden, el área de Gestão do conhecimento (GT4) se revela como otro de los campos de interés en términos del caudal de investigación observado en esta muestra. Fuertemente ligado a la Gestão da Informaçãoy al concepto de Inteligência Competitiva este agrupamiento se establece teniendo en cuenta una débil vinculación 
con el resto de la red, con límites propios, es decir, como un espacio nuevo. Por lo general las temáticas principales giran en torno a los modelos de gestión que pueden aplicarse a diferentes tipos de organizaciones y actividades, no sólo las bibliotecas, abarcando un gran abanico de tópicos que atraviesan el campo de la información y el conocimiento. La interpretación más ajustada es la de estar en presencia de un campo de especialización emergente de características interdisciplinarias. Un buen ejemplo del creciente espacio que ha ganado esta orientación es la reciente aparición la revista Perspectivas em Gestão \& Conhecimento, íntegramente abocada a estas temáticas.

La posición que ocupa el nodo Internet (GT8) es probablemente la más particular de todas dado que se trata de un concepto demasiado amplio y que ejerce un alto grado de intermediación en la red. En principio los vínculos de mayor peso se establecen con Ciência da Informação y Informação en una relación basada en los "espacios comunes" que comparten con ambas temáticas, sobre todo desde ciertos abordajes teóricos. La relación más específica se manifiesta con los nodos Biblioteca digital e Hipertexto, temas que al parecer son los que más comúnmente se manifiestan en las investigaciones que involucran a la red. Sin embargo estas apreciaciones no alcanzan para explicar la posición destacada de este nodo en la red. Un argumento posible es que este concepto es utilizado, en muchos artículos, como sinónimo de tecnología o tecnologías de la información y abordado o vinculado desde muchos aspectos y, por lo tanto, transversal a cualquier área temática. Esto hace que no exista necesariamente un tópico o tópicos delimitados desde dónde se transite el espacio "Internet" desde el punto de vista de la investigación, sino más bien un tema recurrente con múltiples abordajes.

También se destacan en la red una serie de nodos que pueden agruparse, sin una centralidad manifiesta bajo el concepto de Organização do Conhecimento (GT2). Aquí aparecen de manera atomizada una serie de tópicos ligados entre sí que responden a una mirada más tradicional dentro de esta área y que se refleja en el concepto de Organização da Informação vinculado a la Indexação, la Terminología y los Linguagem Documentária. Las nuevas tendencias, en cambio, parecen concentrarse bajo el concepto Representação do Conhecimento con una marcada vinculación al campo de las Ontologías.

Por último, haremos mención de dos temáticas que aparecen en la red y que suelen ser campos de abordaje más comunes o recurrentes en la investigación. El primero de ellos es el relativo a la cuestión profesional que aquí aparece corporizado bajo los conceptos Profissionais da Informação y Bibliotecário con un grado de centralidad importantey, fundamentalmente, una alta intermediación, lo cual indica que es una temática que todavía guarda interés cada vez que se transita aspectos concernientes a la disciplina o al campo de la información.

El segundo espacio temático, el de las bibliotecas, aparece, por el contrario, en un segundo plano. Observando la red puede apreciarse que no constituye un eje importante de la actividad investigadora ya que surge por lo general como un tópico vinculado a un tema mayor. Las Bibliotecas Universitárias emergen, en este sentido, como el segmento más importante dentro de la tipología de Unidades de Información. Finalmente, el proceso de digitalización de las bibliotecas adquiere cierto relieve desde la perspectiva de la aplicación de tecnologías y de los modos de acceso a la información.

Se ha observado además el desempeño que han tenido los principales frentes de investigación a lo largo del período estudiado tal como se muestra en la Figura 2:

Como puede apreciarse en la gráfica existen temas que han mantenido a lo largo de estos diez años una performance bastante lineal respecto al interés que despiertan, como son la Comunicação Científica, la Gestão do Conhecimento e Internet. Otros en cambio presentan fluctuaciones importantes como es el caso de Sociedade da Informação que ha tenido un pico de interés en el año 2000 y que tiene su explicación, como se mencionó líneas atrás, en la sanción en ese año de políticas públicas en Brasil sobre esta cuestión y que obtuvo un alto impacto en la investigación. Sin embargo y de acuerdo a las frecuencias observadas rápidamente decae este interés pasando a ser un tópico de muy escaso abordaje en los años siguientes. Por último, la mayor particularidad se da en las dos áreas que presentan mayor centralidad, Ciência da Informação e Informação, con un comportamiento irregular, de mucha alternancia, lo cual indica que, aún siendo las dos vertientes de la disciplina más investigadas, no responden a una lógica particular o no se pueden
231

TransInformação, Campinas, 25(3):225-235, set./dez., 2013 


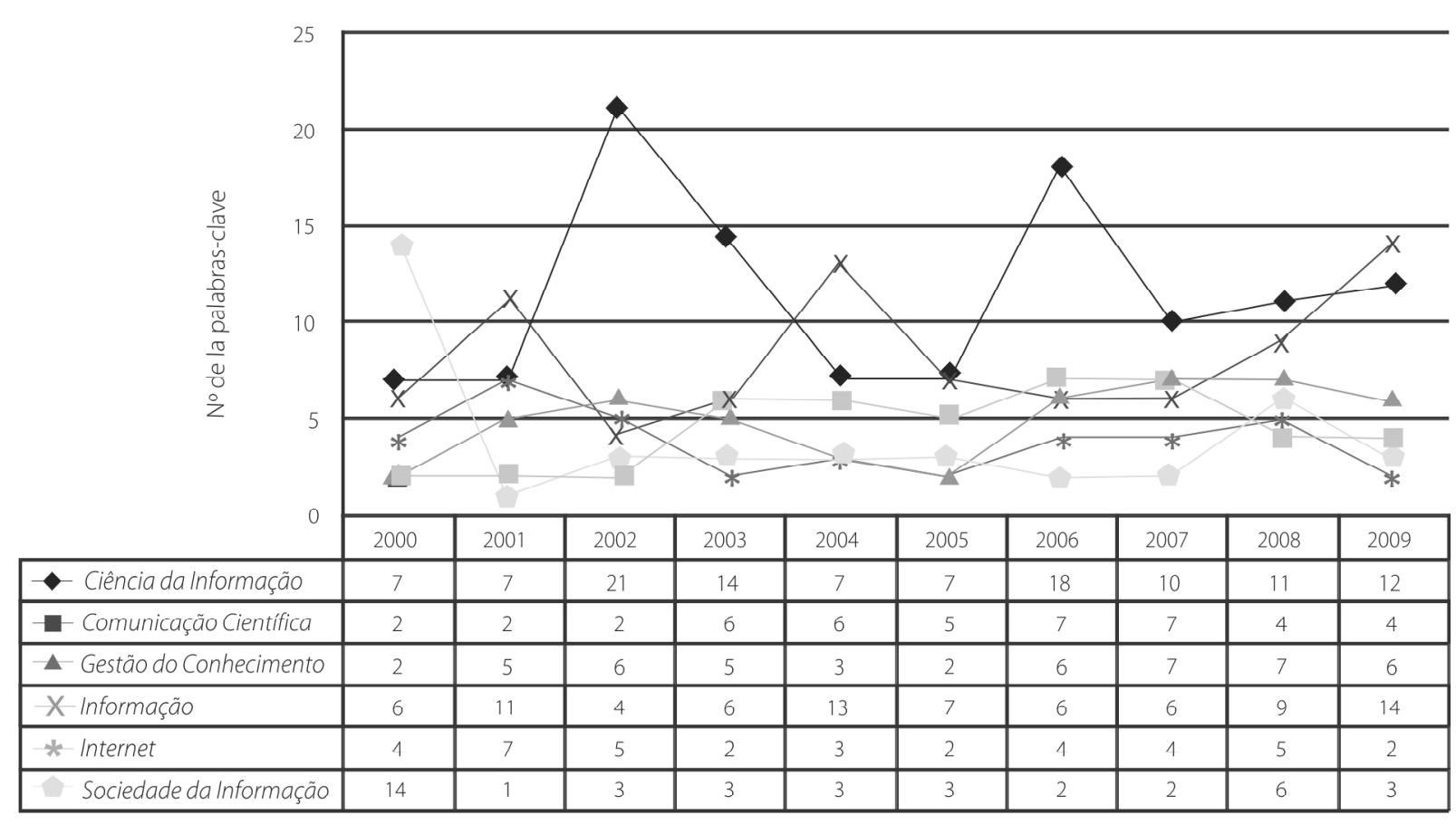

Figura 2. Distribución temporal de las frecuencias de los temas con mayor centralidad.

Fuente: Elaboración propia (2012).

incluir en esta descripción dentro de un argumento más concluyente. Probablemente haya que indagar en otros aspectos de índole contextual - que exceden este trabajo -, para arribar a una explicación más abarcativa.

Al equiparar los frentes de investigación con los grupos de trabajo de la ANCIB se observó una clara correspondencia entre ellos (Tabla 3). De los diez grupos constitutivos señalados por esta organización fueron encontradas equivalencias con siete de ellos. Esto es muy significativo puesto que señala que los espacios institucionales de discusión y consenso proponen una visión real de la disciplina desde el punto de vista de su constitución y estructura, al menos dentro del universo analizado. Al mismo tiempo resulta funcional como matriz de análisis e interpretación de la Ciencia de la Información en Brasil. No obstante ello debe considerarse que esta división puede presentar algunas zonas grises en cuanto a la división de las áreas que se propone teniendo en cuenta la definición explícita que se expone de cada una de ellas. Esto se hizo evidente en los temas que fueron englobados en el GT1 ya que se plantearon dudas acerca de algunos tópicos que, dependiendo del enfoque o la interpretación, pueden formar parte del GT3. También resulta interesante el análisis del GT7 ligado a los aspectos relativos a la producción y comunicación científica, entre los que se encuentran los estudios provenientes del campo bibliométrico. Según se muestra en la red estos aspectos aparecen relacionados aunque las investigaciones basadas en la aplicación del método bibliométrico, en cualquiera de sus variantes, parecen constituir un agrupamiento más autónomo, con un valor de intermediación reducido, lo que le otorga una relativa independencia como espacio de investigación.

Al comparar los resultados de esta muestra con estudios similares realizados en los últimos años pueden apreciarse similitudes en relación a la proporción que guardan las áreas temáticas entre sí respecto del volumen total de investigación publicada. Las comparaciones entre este tipo de trabajos presentan el inconveniente de que las metodologías aplicadas en cada uno de ellos son diferentes $y$, por lo tanto, las conclusiones que pueden extraerse son parciales.

Hecha la salvedad de la cuestión metodológica, la comparativa permite extraer no obstante algunas consideraciones de interés. Una de ellas es que la Ciência da Informação en tanto espacio de estudio disciplinar ha mantenido un interés casi constante en las últimas dos 
Tabla 3. Equivalencias entre las áreas temáticas de ANCIB y los nodos de la red.

\begin{tabular}{|c|c|c|}
\hline Grupo temático & Delimitación temática & Nodos equivalentes en la red \\
\hline GT1 & $\begin{array}{l}\text { Estudos Históricos e Epistemológicos da Ciência da } \\
\text { Informação. }\end{array}$ & $\begin{array}{l}\text { Ciência da Informação, Epistemologia, Interdisciplinaridade, } \\
\text { Ensino, Pesquisa, Biblioteconomia, Produção Científica. }\end{array}$ \\
\hline GT2 & Organização e Representação do Conhecimento. & $\begin{array}{l}\text { Organização do Conhecimento, Organização da Informação, } \\
\text { Linguagem documentária, Terminologia, Indexação, Onto- } \\
\text { logias, Representação do Conhecimento, Recuperação da } \\
\text { Informação. }\end{array}$ \\
\hline GT4 & $\begin{array}{l}\text { Gestão da Informação e do Conhecimento nas Orga- } \\
\text { nizações. }\end{array}$ & $\begin{array}{l}\text { Gestão do Conhecimento, Gestão da Informação, Inteligência } \\
\text { Competitiva, Sistemas de Informação. }\end{array}$ \\
\hline GT5 & Política e Economia da Informação. & $\begin{array}{l}\text { Sociedade da Informação, Sociedade do Conhecimento, } \\
\text { Disseminação da Informação, Inclusão digital, Competência } \\
\text { Informacional, Globalização, Cidadania. }\end{array}$ \\
\hline GT6 & Informação, Educação e Trabalho. & $\begin{array}{l}\text { Informação, Conhecimento, Comunicação, Educação, Tecno- } \\
\text { logia. }\end{array}$ \\
\hline GT7 & $\begin{array}{l}\text { Produção e Comunicação da Informação em Ciência, } \\
\text { Tecnologia e Informação. }\end{array}$ & $\begin{array}{l}\text { Comunicação Científica, Fontes de informação, Periódicos } \\
\text { Eletrônicos, Acesso libre, Arquivos abertos, Avaliação, Bibliotecas } \\
\text { digitais, Bibliometria, Cienciometria, Informetria }\end{array}$ \\
\hline GT8 & Informação e Tecnologia. & Internet, Biblioteca Digital, Ciberespaço, Hipertexto. \\
\hline
\end{tabular}

Nota: ANCIB: Associação Nacional de Pesquisa e Pós-Graduação em Ciência da Informação.

Fuente: Elaboración propia (2012).

décadas, al igual que los aspectos relativos a la Comunicação Científica, la Organização da Conhecimento y las Tecnologias da Informação. Se comprueba que la temática Sociedade da Informação ha tenido un pico de interés dentro de un contexto particular y que su importancia ha ido decayendo en el tiempo. Finalmente la Gestão do Conhecimento/Informação aparece como un área de especialización con un fuerte crecimiento en la década del noventa a tono con la irrupción de las teorías del management en la Ciencia de la Información durante esos años estabilizándose luego en valores más regulares.

\section{Consideraciones Finales}

La delimitación de un campo de conocimiento a través del análisis de los productos intelectuales que genera es una línea de investigación que ha venido consolidándose en el tiempo con un alto grado de aceptación dentro de la comunidad científica. Los resultados de este tipo de estudios han logrado reflejar con bastante precisión y justeza instantáneas de situaciones y escenarios atravesados por múltiples variables constituyéndose además en una herramienta confiable de cara a la evaluación y observación de las tendencias predominantes. Es, al mismo tiempo, un complemento de los constructos teóricos que sustentan las bases de una disciplina en permanente cambio y un medio para contrastar las hipótesis más esenciales acerca de su estructura, contornos y estatus científico. En cierto sentido, esta investigación recoge ambas visiones, esto es, aplicar el método bibliométrico para adentrarse en los perfiles temáticos más salientes de la investigación en Ciencia de la Información e intentar contrastar estos resultados con los postulados vigentes de una organización que corporiza y discute en la actualidad las actividades científicas/académicas de esta disciplina en Brasil como lo es la ANCIB.

Los resultados obtenidos en este trabajo han mostrado que la Ciencia de la Información en Brasil se estructura en la actualidad en siete grandes frentes de investigación con una gran conexidad o interrelación entre algunos de ellos. El dato más saliente de la red calculada evidencia que es la propia disciplina la que ocupa principalmente el debate en el campo científico (desde aspectos teóricos, metodológicos, de formación etc.). Esto explica a nuestro entender por qué el nodo Ciência da Informação adquiere una centralidad abrumadora y con el grado mayor de intermediación. Esta valoración se ve corroborada al comparar los resultados que arrojan estudios similares en donde el 
volumen de artículos publicados sobre esta temática ocupa un lugar preponderante. También la comparativa reafirmó la idea de que algunos tópicos están sujetos a influencias del contexto - político y social -, como es el caso de la Sociedade da Informação que ha tenido una vigencia transitoria en una época en la que Brasil generó cambios importantes en esta materia. Algo similar se observa en el área de la Gestão do Conhecimento/Informação aunque en este caso se trata de una influencia proveniente de nuevos abordajes teóricos, de corte interdisciplinario, de notable auge en los años 1990. Las Tecnologias da Informação han mantenido un interés constante en la investigación pero con algunos matices de época. Durante gran parte de la década del 1990 los tópicos principales giraban en torno a los procesos de automatización de las bibliotecas, mientras que a finales de ésta y a lo largo de los últimos años el entorno digital generado a partir de la irrupción de Internet ha concentrado los mayores esfuerzos de investigación. No obstante ello esta temática resulta a veces de difícil delimitación ya que atraviesa a casi todos los frentes.

El otro punto saliente de este trabajo es la constatación de que los grandes temas que han podido identificarse desde la producción científica tienen una equivalencia directa con la forma en que la ANCIB estructura la disciplina a través de los grupos de trabajo existentes. Este aspecto resulta muy importante no solo desde el punto de vista de la coherencia interna que presenta la Ciencia de la Información en Brasil sino también del enorme avance que esto significa en términos de su institucionalización científica.

\section{Referencias}

ARAÚJO, C.A.A.; MELO, M.O.T. Análise dos quinze anos do periódico Perspectivas em Ciência da Informação. Perspectivas em Ciência da Informação, v. 16, n.4, p.243-256, 2011.

DELGADO-LÓPEZ-CÓZAR, E. Lainvestigación en biblioteconomía y documentación. Gijón: Trea, 2002.

ELIEL, R.A. Institucionalização da ciência da informação no Brasil: estudo da convergencia entre a produção científica e os marcos regulatórios da área. Transinformação, v.20, n.3, p.207-224, 2008.

FRANCELIN, M.M. Configuração epistemológica da ciência da informação no Brasil em uma perspectiva pós-moderna: análise de periódicos da área. Ciência da Informação, v.33, n.2, p.49-66, 2004.

FREIRE, I.M. Um olhar sobre a produção científica brasileira na temática epistemologia da ciência da informação. Tendências da Pesquisa Brasileira em Ciência da Informação, v.1, n.1, p.2-31, 2008

HANNEMAN, R.; RIDDLE, M. Introduction to social network methods. Riverside: University of California, 2005. Available from: <http://faculty.ucr.edu/ hanneman/>. Cited: May 24, 2012.

HERRERO-SOLANA, V.; LIBERATORE, G. Visibilidad internacional de las revistas iberoamericanas de bibliotecología y documentación. Revista Española de Documentación Científica, v.31, n.2, p.230-239, 2008.

HERRERO-SOLANA, V.; MIGUEL, S. Visibilidad de las revistas latinoamericanas de bibliotecología y ciencia de la información a través de Google Scholar. Ciência da Informação, v.39, n.2, p.54-67, 2010.

HERRERO-SOLANA, V.; RÍOS-GÓMEZ, C. Producción latinoamericana en biblioteconomía y documentación en el
Social Science Citation Index (SSCI) 1966-2003. Information Research, v.11, n.2, 2006. Disponible en: <http://informationr. net/ir/11-2/paper247.html>. Acceso en: 22 mar. 2012.

JARVELIN, K.; VAKKARI, P. Content analysis of research articles in library and information science. Library and Information Science Research, v.12, p.392-421, 1990.

KAMADA, T.; KAWAI, S. An algorithm for drawing general undirected graphs. Information Processing Letters, v.31, n.1, p.7-15, 1989.

KOBASHI, N.Y. Estudos de institucionalização social e cognitiva da pesquisa científica no Brasil: reflexões sobre um programa de pesquisa. In: LARA, M.L.; FUJINO, A.; NORONHA, D.P. Informação e contemporaneidade: perpectivas. Recife: Néctar, 2007. p.191-204.

LIBERATORE, G.; HERRERO-SOLANA, V.; GUIMARAES, J.A.C. Análisis bibliométrico de la revista brasileña Ciência da Informação. Brazilian Journal of Information Science, v.1, n.2, p.3-21, 2007.

LICEA DE ARENAS, J. et al. Una visión bibliométrica de la investigación en bibliotecología y ciencia de la información en América Latina y el Caribe. Revista Española de Documentación Científica, v.23, n.1, p.45-53, 2000.

MALTRÁS-BARBA, B. Los indicadores bibliométricos:fundamentos y aplicación al análisis de la ciencia. Gijón: TREA, 2003.

MENDONÇA, E.S. A lingüística e a ciência da informação: estudos de uma interseção. Ciência da Informação, v.29, n.3, p.50-70, 2000.

MIGUEL, S.; MOYA-ANEGÓN, F.; HERRERO-SOLANA, V. EI análisis de co-citas como método de investigación en bibliotecología y ciencia de la información. Investigación Bibliotecológica, v.21, n.43, p.139-155, 2007. 
MIRANDA, A.; BARRETO, A.A. Pesquisa em ciência da informação no Brasil: síntese e perspectiva. DataGramaZero: Revista deCiência da Informação, v.1, n.6, 2000. Disponível em: <http:/ /dgz.org.br>. Acesso em: 15 abr. 2012.

MOYA-ANEGÓN, F.; HERRERO-SOLANA, V. Visibilidad internacional de la producción científica iberoamericana en biblioteconomía y documentación (1991-2000). Ciência da Informação, v.31, n.3, p.54-65, 2002.

MUELLER, S.P.M.; MIRANDA, A.; SUAIDEN, E.J. A pesquisa em ciência da informação no Brasil: análise dos trabalhos apresentados no IV Enancib, Brasília, 2000. Revista de Biblioteconomia de Brasília, v.23/24, n.3, p.293-308, 2000.

MUELLER, S.P.M.; PECEGUEIRO, C.M.P.A. O periódico Ciência da Informação na década de 90: um retrato da área refletido em seus artigos. Ciência da Informação, v.30, n.2, p.47-63, 2001.

NORONHA, D.P.; POBLACIÓN, D.A. Producción académica de docentes/doctores de los programas de posgrado en ciencia de la información en Brasil. Ciencias de la Información, v.33, n.1, p.25-33, 2002

PARREIRAS, F.S.; SILVA, A.B.O.; MATHEUS, R.F. RedeCl: colaboração e produção científica em ciência da informação no Brasil. Perspectivas em Ciência da Informação, v.11, n.3, p.302-317, 2006.

PECEGUEIRO, C.M.P.A. Temática dos artigos de periódicos brasileiros na área da ciência da informação na década de 90 . Transinformação, v.14, n.2, p.117-131, 2002.

PINHEIRO, L.V.R. Infra-estrutura da pesquisa em ciência da informação no Brasil. DataGramaZero: Revista de Ciência da Informação, v.1, n.6, 2000. Disponível em: <http://dgz.org.br>. Acesso em: 15 abr. 2012.
PINHEIRO, L.V.R. Evolução e tendências da ciência da informação, no exterior e no Brasil. In: ENCONTRO NACIONAL DE PESQUISA EM CIÊNCIA DA INFORMAÇÃO, 6., 2005, Florianópolis. Anais... Florianópolis: Ancib, 2005. p.12.

PINHEIRO, L.V.R.; BRÄSCHER, M.; BURNIER, S. Ciência da informação: 32 anos (1972-2004) no caminho da história e horizontes de um periódico científico brasileiro. Ciência da Informação, v.34, n.3, p.23-75, 2005.

PINHEIRO, L.V.R.; LOUREIRO, J.M.M. Políticas públicas de C\&T, ICT e de pós-graduação em abordagem histórico-cultural da ciência da informação no Brasil. In: ENCONTRO NACIONAL DE CIÊNCIA DA INFORMAÇÃO, 5., 2004, Salvador. Anais eletrônicos... Salvador: Cinfor, 2004. Disponível em: <http:// www.cinform.ufba.br/v_anais/frames.html>. Acesso em: 10 abr. 2012.

PINTO, A.L.; MOREIRO-GONZÁLEZ, J.A. Representación productiva de los programas de postgrado en biblioteconomía y ciencia de la Información en Brasil: visibilidad en las bases de datos y aspectos del currículo Lattes de los investigadores. Em Questão, v.16, n.2, p.15-32, 2010.

SILVA, F.M. Análise da revista Ciência da Informação disponibilizada na SciELO a partir do seu vocabulário controlado. Transinformação, v.14, n.2, p.133-138, 2002.

VARGAS-QUESADA, B.M.; CHINCHILLA-RODRÍGUEZ, Z.; MOYA-ANEGÓN, F. Estructura de la colaboración científica española en biblioteconomía y documentación (Scopus 1999-2007). Revista Interamericana de Bibliotecología, v.33, n.1, p.105-123, 2010.

VIEIRA, K.C. Temas enfocados em Transinformação de 1989 a 1996. In: WITTER, G.P. (Org.). Produção científica. Campinas: Átomo, 1997. p.41-54. 

